\title{
Existence of Nonnegative Solutions for Fourth Order Elliptic Equations of Kirchhoff Type with General Subcritical Growth
}

\author{
Jianping Huang and Qi Zhang*
}

\begin{abstract}
This paper is dedicated to investigating the following fourth-order elliptic equation with Kirchhoff-type

$$
\left\{\begin{array}{l}
\Delta^{2} u-\left(a+b \int_{\mathbb{R}^{N}}|\nabla u|^{2} d x\right) \Delta u+c u=f(u) \text { in } \mathbb{R}^{N}, \\
u \in H^{2}\left(\mathbb{R}^{N}\right),
\end{array}\right.
$$

where $a>0, b \geq 0$ and $c>0$ are constants. By using cut-off functional and monotonicity tricks, we prove that the above problem has a positive solution. Our result cover the case where the nonlinearity satisfies asymptotically linear and superlinear condition at the infinity, which extend the results of related literatures.
\end{abstract}

\section{Introduction}

In this paper, we consider the following fourth order elliptic equation of Kirchhoff type:

$$
\left\{\begin{array}{l}
\Delta^{2} u-\left(a+b \int_{\mathbb{R}^{N}}|\nabla u|^{2} d x\right) \Delta u+c u=f(u) \text { in } \mathbb{R}^{N}, \\
u \in H^{2}\left(\mathbb{R}^{N}\right),
\end{array}\right.
$$

where $N \geq 5, \Delta^{2}:=\Delta(\Delta)$ is the biharmonic operator, $a, b, c$ are positive constants and $f: \mathbb{R} \rightarrow \mathbb{R}$ satisfies

$\left(\mathrm{f}_{1}\right) f \in \mathcal{C}\left(\mathbb{R}_{+}, \mathbb{R}_{+}\right)$with $\mathbb{R}_{+}=[0,+\infty)$, and $\lim _{t \rightarrow 0^{+}} f(t) / t=0 ;$

$\left(\mathrm{f}_{2}\right) \lim _{t \rightarrow+\infty} f(t) / t^{2 * *}-1=0$ with $2_{* *}=2 N /(N-4) ;$

$\left(\mathrm{f}_{3}\right)$ there exists $\tau>0$ such that $F(\tau)>\frac{c}{2} \tau^{2}$, where $F(\tau)=\int_{0}^{\tau} f(s) d s$.

The problem (1.1) is related to the stationary analogue of the evolution of the equation of Kirchhoff type

$$
u_{t t}+\Delta^{2} u-\left(a+b \int_{\mathbb{R}^{N}}|\nabla u|^{2} d x\right) \Delta u=g(x, u),
$$

Received September 12, 2017; Accepted February 17, 2019.

Communicated by Jann-Long Chern.

2010 Mathematics Subject Classification. 35J35, 35J50, 35J60.

Key words and phrases. fourth order elliptic equations, cut-off functional, Pohožaev equality.

*Corresponding author. 
which is used to describe some phenomena appeared in physics and engineering, due to it is regarded as a good approximation for describing vibrations of beams or plates, see [1,2]. Obviously, the problem (1.1) is a nonlocal problem because of the appearance of the term $b \int_{\mathbb{R}^{N}}|\nabla u|^{2} d x$ which causes some mathematical difficulties. In the same time, this provokes the problem more interesting.

On the one hand, for the general case of the problem (1.1) with $c=V(x)$, then this problem is reduced to the following fourth-order elliptic equation of Kirchhoff type

$$
\left\{\begin{array}{l}
\Delta^{2} u-\left(a+b \int_{\mathbb{R}^{N}}|\nabla u|^{2} d x\right) \Delta u+V(x) u=g(x, u) \text { in } \mathbb{R}^{N}, \\
u \in H^{2}\left(\mathbb{R}^{N}\right) .
\end{array}\right.
$$

With the aid of variational methods, under various conditions of the potential $V(x)$ and the nonlinearity $g(x, u)$, some valuable results for the problem $(1.2)$ have been wide range of investigated in the literature. For example, Zhang et al. 29] studied the existence of the least energy sign-changing solution for the problem $(1.2)$ by constraint variational method and quantitative deformation lemma. Xu and Chen [23] dealt with problem (1.2) with supcubic case, and the existence of infinitely many solutions for the problem is established by using symmetric mountain pass theorem. For related works, one can see $11,12,16|18,21| 22$ and the references therein.

There are also some works on the existence and multiplicity results for following elliptic equation

$$
\begin{cases}\Delta^{2} u-\left(a+b \int_{\mathbb{R}^{N}}|\nabla u|^{2} d x\right) \Delta u=g(x, u) & \text { in } \Omega, \\ u=\Delta u=0 & \text { on } \partial \Omega,\end{cases}
$$

where $\Omega \subset \mathbb{R}^{N}$ is a bounded domain with a smooth boundary. More precisely, in [19], the authors using the mountain pass techniques and the truncation method studied the existence of nontrivial solutions for the problem (1.3). In recent paper [17], Song and Shi did with problem 1.3 with critical exponent case, the new results about the existence and multiplicity of solutions for the problem are obtained by using the concentration compactness principle and variational method. For more results related to elliptic equations of Kirchhoff type, we refer the readers to $3,7,2,14,26,28]$ and the references therein.

We also note that the problem (1.1) with $a \equiv 1$ and $b \equiv 0$ has been studied for the nonlinear biharmonic equation and system, which problem arises in the study of travelling waves in suspension bridge (see [15]) and the study of the static deflection of an elastic plate in a fluid. There are many works, see [13, 20, 24, 25, 27, 30]. In particular, Zhang [30] and Ye [24] studied the sublinear case; 27] and [25] studied the super-quadratic case. Here, we do not try to review the huge bibliography. 
Inspired by the works mentioned above, in the present paper, our aim is to establish the existence of nonnegative solutions for the problem 1.1) with the nonlinearity $f$ asymptotically linear and superlinear at the infinity. Under some weaker assumption, we reach the goal by using cut-off function and monotonicity tricks. To the best of our knowledge, there has no works concerning this case up to now. Comparing the aforementioned results, our result is different and extends them to some extent.

Since we are looking for positive solutions to the problem (1.1), without loss of generality, we suppose that $f(t)=0$ for all $t<0$.

Now, we are ready to state the main results of this paper.

Theorem 1.1. Assume that $N \geq 5, a, b, c$ are positive constants. Suppose that $\left(\mathrm{f}_{1}\right)-\left(\mathrm{f}_{3}\right)$ hold. Then there exists $b_{0}>0$ such that for any $b \in\left[0, b_{0}\right)$, the problem (1.1) has a nonnegative solution.

The conditions $\left(f_{2}\right)$ and $\left(f_{3}\right)$ are sometimes replaced by the following stronger asymptotically linear assumption.

$\left(\mathrm{f}_{4}\right) \lim _{t \rightarrow+\infty} f(t) / t=\Theta$, where $\Theta \in(c,+\infty)$.

To show that the condition $\left(f_{4}\right)$ implies $\left(f_{2}\right)$ and $\left(f_{3}\right)$ is easy. Indeed, from $\left(f_{4}\right)$, one has

$$
\lim _{t \rightarrow+\infty} \frac{f(t)}{t^{2 * *}-1}=\lim _{t \rightarrow+\infty} \frac{f(t)}{t t^{2 * *}-2}=0,
$$

which shows that $\left(f_{2}\right)$ holds. On the other hand, from the condition $\left(f_{4}\right)$, there exists a constant $K>0$ such that for any $t>K$,

$$
\frac{\Theta+c}{2} t<f(t)
$$

Thus,

$$
F(t)=\int_{0}^{t} f(s) d s=\int_{0}^{K} f(s) d s+\int_{K}^{t} f(s) d s \geq \int_{K}^{t} f(s) d s>\frac{\Theta+c}{4}\left(t^{2}-K^{2}\right),
$$

and if only $t \geq K\left(\frac{\Theta+c}{\Theta-c}\right)^{1 / 2}$, there holds $F(t) \geq \frac{c}{2} t^{2}$, which implies that $\left(\mathrm{f}_{3}\right)$ holds. Consequently, we have the following corollary right away.

Corollary 1.2. Assume that $N \geq 5, a, b, c$ are positive constants. Suppose that $\left(\mathrm{f}_{1}\right)$ and $\left(\mathrm{f}_{4}\right)$ hold. Then there exists $b_{0}>0$ such that for any $b \in\left[0, b_{0}\right)$, the problem (1.1) has a nonnegative solution.

Notations. Throughout this paper we make use of the following notations:

- $H^{2}\left(\mathbb{R}^{N}\right)$ denotes the usual Hilbert space; 
- For $1 \leq s<\infty, L^{s}\left(\mathbb{R}^{N}\right)$ denotes the Lebesgue space with the norm $\|u\|_{s}=$ $\left(\int_{\mathbb{R}^{N}}|u|^{s} d x\right)^{1 / s}$

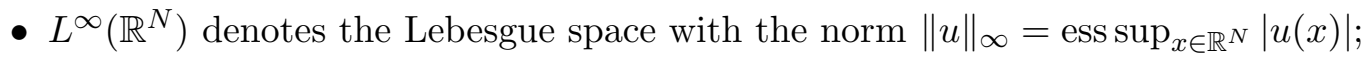

- $X^{-1}$ denotes the dual space of a Banach space $X$;

- $\langle\cdot, \cdot\rangle$ denotes the duality pairing between $X^{-1}$ and $X$;

- meas $(\cdot)$ denotes the Lebesgue measure in $\mathbb{R}^{N}$;

- For any $x \in \mathbb{R}^{N}$ and $r>0, B_{r}(x):=\left\{y \in \mathbb{R}^{N}:|x-y|<r\right\}$;

- $C_{1}, C_{2}, C_{3}, C_{4}, C_{5}$ denote positive constants possibly different in different places.

The rest of the paper is organized as follows. In Section 2, some preliminary results are presented. Section 3 is devoted to finding a nonnegative solution for the problem (1.1).

\section{Preliminaries}

Because equation 1.1 is autonomous, according to the symmetric critical principle, any critical point $u \in H_{r}^{2}\left(\mathbb{R}^{N}\right)$ is also a critical point of $H^{2}\left(\mathbb{R}^{N}\right)$. Hence to seek weak solutions of problem 1.1 , we need only discuss in $H_{r}^{2}\left(\mathbb{R}^{N}\right)$. Let

$$
E:=\left\{u \in H^{2}\left(\mathbb{R}^{N}\right) \mid u \text { is radially symmetric }\right\},
$$

equipped with the inner product

$$
(u, v)=\int_{\mathbb{R}^{N}}(\Delta u \Delta v+a \nabla u \nabla v+c u v) d x, \quad \forall u, v \in E
$$

and the induced norm $\|u\|=(u, u)^{1 / 2}$. Obviously, $E$ is a Hilbert space. And the embed$\operatorname{ding} E \hookrightarrow L^{s}\left(\mathbb{R}^{N}\right)$ is continuous for $2 \leq s \leq 2_{* *}$, that is, there exists $\gamma_{s}$ such that

$$
\|u\|_{s} \leq \gamma_{s}\|u\|, \quad \forall u \in E
$$

Furthermore, the embedding $E \hookrightarrow L^{s}\left(\mathbb{R}^{N}\right)$ is compact for $s \in\left(2,2_{* *}\right)$.

The energy functional $\mathcal{J}_{b}$ related to the problem (1.1) on $E$ is

$$
\mathcal{J}_{b}(u)=\frac{1}{2}\|u\|^{2}+\frac{b}{4}\left(\int_{\mathbb{R}^{N}}|\nabla u|^{2} d x\right)^{2}-\int_{\mathbb{R}^{N}} F(u) d x .
$$

From the conditions $\left(\mathrm{f}_{1}\right)$ and $\left(\mathrm{f}_{2}\right)$, for any $\varepsilon>0$, there exists $C_{\varepsilon}>0$ such that

$$
F(t) \leq \varepsilon t^{2}+C_{\varepsilon} t^{2 * *}, \quad \forall t \in \mathbb{R}_{+} .
$$


Consequently, the functional $\mathcal{J}_{b}$ is well defined and is of class $\mathcal{C}^{1}(E, \mathbb{R})$. Moreover, for any $u, v \in E$, one has

$$
\left\langle\mathcal{J}_{b}^{\prime}(u), v\right\rangle=(u, v)+b \int_{\mathbb{R}^{N}}|\nabla u|^{2} d x \int_{\mathbb{R}^{N}} \nabla u \nabla v d x-\int_{\mathbb{R}^{N}} f(u) v d x .
$$

In order to find a bounded (PS) sequence for the functional $\mathcal{J}_{b}$, we adopt the method introduced by 9,10 . Hence we introduce the modified functional $\mathcal{J}_{b, \lambda}^{T}: E \rightarrow \mathbb{R}$

$$
\mathcal{J}_{b, \lambda}^{T}(u)=\frac{1}{2}\|u\|^{2}+\frac{b}{4} h_{T}(u)\left(\int_{\mathbb{R}^{N}}|\nabla u|^{2} d x\right)^{2}-\lambda \int_{\mathbb{R}^{N}} F(u) d x
$$

of $\mathcal{J}_{b}$, where $\lambda>0$ and $h_{T}(u)=\psi\left(\|u\|^{2} / T^{2}\right)$ for any $T>0$, with the cut-off function $\psi \in \mathcal{C}^{\infty}\left(\mathbb{R}_{+}, \mathbb{R}_{+}\right)$satisfying

$$
\begin{cases}\psi(t)=1 & \text { if } t \in[0,1], \\ 0 \leq \psi(t) \leq 1 & \text { if } t \in(1,2), \\ \psi(t)=0 & \text { if } t \in[2,+\infty), \\ \left\|\psi^{\prime}\right\|_{\infty} \leq 2 . & \end{cases}
$$

Now, to find a critical point $u$ of $\mathcal{J}_{b}$, we have to verify that the critical point of $\mathcal{J}_{b}^{T}$ satisfies $\|u\| \leq T$. To proceed we first present a useful proposition which developed by Jeanjean.

Proposition 2.1. (see [8, Theorem 1.1]) Let $X$ be a Banach space equipped with a norm $\|\cdot\|_{X}$ and let $I \subset \mathbb{R}_{+}$be an interval. We consider a family $\left\{\Phi_{\lambda}\right\}_{\lambda \in I}$ of $\mathcal{C}^{1}$-functional on $X$ of the norm

$$
\Phi_{\lambda}(u)=A(u)-\lambda B(u), \quad \forall \lambda \in I,
$$

where $B(u) \geq 0$ for all $u \in X$, and such that either $A(u) \rightarrow+\infty$ or $B(u) \rightarrow+\infty$, as $\|u\|_{X} \rightarrow+\infty$. We assume that there are two points $v_{1}, v_{2}$ in $X$ such that

$$
c_{\lambda}:=\inf _{\gamma \in \Gamma} \max _{t \in[0,1]} \Phi_{\lambda}(\gamma(t))>\max \left\{\Phi_{\lambda}\left(v_{1}\right), \Phi_{\lambda}\left(v_{2}\right)\right\}
$$

where

$$
\Gamma=\left\{\gamma \in \mathcal{C}([0,1], X): \gamma(0)=v_{1}, \gamma(1)=v_{2}\right\}
$$

Then, for almost every $\lambda \in I$, there exists a sequence $\left\{u_{n}\right\} \subset X$ such that

(i) $\left\{u_{n}\right\}$ is bounded in $X$;

(ii) $\Phi_{\lambda}\left(u_{n}\right) \rightarrow c_{\lambda}$;

(iii) $\Phi_{\lambda}^{\prime}\left(u_{n}\right) \rightarrow 0$ in $X^{-1}$. 
By Proposition 2.1, in our case, $X=E, \Phi_{\lambda}=\mathcal{J}_{b, \lambda}^{T}, v_{1}=0, v_{2}$ will be found in Section 3 ,

$$
A(u)=\frac{1}{2}\|u\|^{2}+\frac{b}{4} h_{T}(u)\left(\int_{\mathbb{R}^{N}}|\nabla u|^{2} d x\right)^{2} \quad \text { and } \quad B(u)=\int_{\mathbb{R}^{N}} F(u) d x .
$$

The perturbed functional is

$$
\Phi_{\lambda}(u)=\mathcal{J}_{b, \lambda}^{T}(u)=\frac{1}{2}\|u\|^{2}+\frac{b}{4} h_{T}(u)\left(\int_{\mathbb{R}^{N}}|\nabla u|^{2} d x\right)^{2}-\lambda \int_{\mathbb{R}^{N}} F(u) d x .
$$

In addition, for any $u, v \in E$,

$$
\begin{aligned}
\left\langle\left(\mathcal{J}_{b, \lambda}^{T}\right)^{\prime}(u), v\right\rangle= & (u, v)+\frac{b}{2 T^{2}} \psi^{\prime}\left(\frac{\|u\|^{2}}{T^{2}}\right)\left(\int_{\mathbb{R}^{N}}|\nabla u|^{2} d x\right)^{2}(u, v) \\
& +b \psi\left(\frac{\|u\|^{2}}{T^{2}}\right) \int_{\mathbb{R}^{N}}|\nabla u|^{2} d x \int_{\mathbb{R}^{N}} \nabla u \nabla v d x-\lambda \int_{\mathbb{R}^{N}} f(u) v d x .
\end{aligned}
$$

To prove the boundedness of (PS) sequence, we need the following Pohožaev identity.

Lemma 2.2. Let $N \geq 5$. If $u \in E$ is a critical point of $\mathcal{J}_{b, \lambda}^{T}$, namely, a week solution of

$$
\left[1+\frac{b}{2 T^{2}} \psi^{\prime}\left(\frac{\|u\|^{2}}{T^{2}}\right)\left(\int_{\mathbb{R}^{N}}|\nabla u|^{2} d x\right)^{2}\right] H u-b \psi\left(\frac{\|u\|^{2}}{T^{2}}\right) \int_{\mathbb{R}^{N}}|\nabla u|^{2} d x \Delta u=\lambda f(u),
$$

where $H u=\Delta^{2} u-a \Delta u+c u$, then the following Pohožaev type identity holds

$$
\begin{aligned}
\lambda N \int_{\mathbb{R}^{N}} F(u) d x \\
=\frac{1}{2}\left[(N-4) \int_{\mathbb{R}^{N}}|\Delta u|^{2} d x+a(N-2) \int_{\mathbb{R}^{N}}|\nabla u|^{2} d x+c N \int_{\mathbb{R}^{N}} u^{2} d x\right] \\
+\frac{b(2 N-4)}{4} \psi\left(\frac{\|u\|^{2}}{T^{2}}\right)\left(\int_{\mathbb{R}^{N}}|\nabla u|^{2} d x\right)^{2} \\
+\frac{b}{4 T^{2}} \psi^{\prime}\left(\frac{\|u\|^{2}}{T^{2}}\right)\left(\int_{\mathbb{R}^{N}}|\nabla u|^{2} d x\right)^{2} \\
\quad \times\left[(N-4) \int_{\mathbb{R}^{N}}|\Delta u|^{2} d x+a(N-2) \int_{\mathbb{R}^{N}}|\nabla u|^{2} d x+c N \int_{\mathbb{R}^{N}} u^{2} d x\right] .
\end{aligned}
$$

Proof. Let $u \in E \backslash\{0\}$. We make a transformation $u_{t}: E \rightarrow E$ satisfying

$$
u_{t}(x)=u\left(\frac{x}{t}\right), \quad t>0 .
$$

Then

$$
\begin{aligned}
\mathcal{J}_{b, \lambda}^{T}\left(u_{t}\right)= & \frac{1}{2}\left[t^{N-4} \int_{\mathbb{R}^{N}}|\Delta u|^{2} d x+a t^{N-2} \int_{\mathbb{R}^{N}}|\nabla u|^{2} d x+t^{N} c \int_{\mathbb{R}^{N}} u^{2} d x\right] \\
& +\frac{b t^{2 N-4}}{4} \psi\left(\frac{\int_{\mathbb{R}^{N}}\left[t^{N-4}|\Delta u|^{2}+a t^{N-2}|\nabla u|^{2}+t^{N} c u{ }^{2}\right] d x}{T^{2}}\right)\left(\int_{\mathbb{R}^{N}}|\nabla u|^{2} d x\right)^{2} \\
& -\lambda t^{N} \int_{\mathbb{R}^{N}} F(u) d x .
\end{aligned}
$$


Since $u$ is a critical point of $\mathcal{J}_{b, \lambda}^{T}$, we can get

$$
\begin{aligned}
0= & \left.\frac{d \mathcal{J}_{b, \lambda}^{T}\left(u_{t}\right)}{d t}\right|_{t=1} \\
= & {\left[(N-4) \int_{\mathbb{R}^{N}}|\Delta u|^{2} d x+a(N-2) \int_{\mathbb{R}^{N}}|\nabla u|^{2} d x+c N \int_{\mathbb{R}^{N}} u^{2} d x\right] } \\
& \times\left[\frac{1}{2}+\frac{b}{4 T^{2}} \psi^{\prime}\left(\frac{\|u\|^{2}}{T^{2}}\right)\left(\int_{\mathbb{R}^{N}}|\nabla u|^{2} d x\right)^{2}\right] \\
& +\frac{b(2 N-4)}{4} \psi\left(\frac{\|u\|^{2}}{T^{2}}\right)\left(\int_{\mathbb{R}^{N}}|\nabla u|^{2} d x\right)^{2}-\lambda N \int_{\mathbb{R}^{N}} F(u) d x .
\end{aligned}
$$

The desired identity (2.3) follows immediately from (2.4).

\section{Proof of the main results}

To prove the main results of the paper, we need the following lemmas.

Lemma 3.1. Suppose that $\left(\mathrm{f}_{1}\right)-\left(\mathrm{f}_{3}\right)$ hold. Then there exist a constant $\sigma \in(0,1)$ and $v_{2} \in E$ such that $\mathcal{J}_{b, \lambda}^{T}\left(v_{2}\right)<0$ for all $\lambda \in I:=[\sigma, 1]$.

Proof. Let $L>0, \tau$ as in $\left(\mathrm{f}_{3}\right)$, define $z: \mathbb{R}^{N} \rightarrow \mathbb{R}$ as

$$
z(x)= \begin{cases}\tau & \text { for }|x| \leq L, \\ \tau(L+1-|x|) & \text { for } L<|x|<L+1, \\ 0 & \text { for }|x| \geq L+1 .\end{cases}
$$

Then $z \in E$, and it follows from $\left(\mathrm{f}_{3}\right)$ that

$$
\begin{aligned}
\int_{\mathbb{R}^{N}}\left(F(z)-\frac{c}{2} z^{2}\right) d x= & \int_{B_{L}}\left(F(z)-\frac{c}{2} z^{2}\right) d x+\int_{B_{L+1} \backslash B_{L}}\left(F(z)-\frac{c}{2} z^{2}\right) d x \\
\geq & \left(F(\tau)-\frac{c}{2} \tau^{2}\right) \operatorname{meas}\left(B_{L}\right) \\
& -\operatorname{meas}\left(B_{L+1}-B_{L}\right)\left(\max _{t \in[0, \tau]}\left|F(t)-\frac{c}{2} t^{2}\right|\right) \\
\geq & C_{1} L^{N}-C_{2} L^{N-1} .
\end{aligned}
$$

From (3.1), for $L>1$ big enough, we have

$$
\int_{\mathbb{R}^{N}}\left(F(z)-\frac{c}{2} z^{2}\right) d x>0
$$

Further, there exists $\sigma \in(0,1)$ such that

$$
\int_{\mathbb{R}^{N}}\left(\sigma F(z)-\frac{c}{2} z^{2}\right) d x>0 .
$$


On the other hand, setting $v_{2}(x)=z\left(x / t_{0}\right)$ with $t_{0}>0$ large enough, one has

$$
\begin{aligned}
\int_{\mathbb{R}^{N}}\left(\sigma F\left(v_{2}\right)-\frac{c}{2} v_{2}^{2}\right) d x & =t_{0}^{N} \int_{\mathbb{R}^{N}}\left(\sigma F(z)-\frac{c}{2} z^{2}\right) d x \\
& >\frac{t_{0}^{N-4}}{2} \int_{\mathbb{R}^{N}}|\Delta z|^{2} d x+\frac{a t_{0}^{N-2}}{2} \int_{\mathbb{R}^{N}}|\nabla z|^{2} d x \\
& =\frac{1}{2} \int_{\mathbb{R}^{N}}\left|\Delta v_{2}\right|^{2} d x+\frac{a}{2} \int_{\mathbb{R}^{N}}\left|\nabla v_{2}\right|^{2} d x
\end{aligned}
$$

and

$$
\left\|v_{2}\right\|^{2}=t_{0}^{N-4} \int_{\mathbb{R}^{N}}|\Delta z|^{2} d x+a t_{0}^{N-2} \int_{\mathbb{R}^{N}}|\nabla z|^{2} d x+c t_{0}^{N} \int_{\mathbb{R}^{N}} z^{2} d x \geq 2 T^{2} .
$$

Thus, by the definition $\mathcal{J}_{b, \lambda}^{T}$, the cut-off function $\psi,(3.2)$ and $(3.3)$, for all $\lambda \in I:=[\sigma, 1]$, we obtain that

$$
\begin{aligned}
\mathcal{J}_{b, \lambda}^{T}\left(v_{2}\right) & =\frac{1}{2}\left\|v_{2}\right\|^{2}-\lambda \int_{\mathbb{R}^{N}} F\left(v_{2}\right) d x \\
& \leq \frac{1}{2}\left\|v_{2}\right\|^{2}-\sigma \int_{\mathbb{R}^{N}} F\left(v_{2}\right) d x \\
& =\frac{1}{2} \int_{\mathbb{R}^{N}}\left(\left|\Delta v_{2}\right|^{2}+a\left|\nabla v_{2}\right|^{2}+c v_{2}^{2}\right) d x-\sigma \int_{\mathbb{R}^{N}} F\left(v_{2}\right) d x \\
& =\frac{1}{2} \int_{\mathbb{R}^{N}}\left(\left|\Delta v_{2}\right|^{2}+a\left|\nabla v_{2}\right|^{2}\right) d x-\int_{\mathbb{R}^{N}}\left(\sigma F\left(v_{2}\right)-\frac{c}{2} v_{2}^{2}\right) d x \\
& <0 .
\end{aligned}
$$

The proof is completed.

Lemma 3.2. Suppose that $\left(\mathrm{f}_{1}\right)-\left(\mathrm{f}_{3}\right)$ hold. Then there exists a constant $c_{0}>0$ such that $c_{\lambda} \geq c_{0}$ for all $\lambda \in I$.

Proof. For all $u \in E$ and $\lambda \in I$, using (2.1) and $(2.2)$ with $\varepsilon=1 /\left(4 \tau_{2}^{2}\right)$, we have

$$
\begin{aligned}
\mathcal{J}_{b, \lambda}^{T}(u) & =\frac{1}{2}\|u\|^{2}+\frac{b}{4} h_{T}(u)\left(\int_{\mathbb{R}^{N}}|\nabla u|^{2} d x\right)^{2}-\lambda \int_{\mathbb{R}^{N}} F(u) d x \\
& \geq \frac{1}{2}\|u\|^{2}-\int_{\mathbb{R}^{N}} F(u) d x \\
& \geq \frac{1}{2}\|u\|^{2}-\varepsilon \int_{\mathbb{R}^{N}} u^{2} d x-C_{\varepsilon} \int_{\mathbb{R}^{N}}|u|^{2 * *} d x \\
& \geq \frac{1}{4}\|u\|^{2}-C_{\varepsilon} \gamma_{2_{* *}^{* *}}^{2_{* *}}\|u\|^{2_{* *}} .
\end{aligned}
$$

Then, there exists $\alpha>0$ such that $\mathcal{J}_{b, \lambda}^{T}(u)>0$ for all $0<\|u\| \leq \alpha$ and any $\lambda \in I$. Particularly, for $\|u\|=\alpha$, there exists $c_{0}>0$ such that $\mathcal{J}_{b, \lambda}^{T}(u) \geq c_{0}$. On the other hand, from Lemma 3.1, we have $\mathcal{J}_{b, \lambda}^{T}(\gamma(1))=\mathcal{J}_{b, \lambda}^{T}\left(v_{2}\right)<0$ for all $\gamma \in \Gamma$. So $\|\gamma(1)\|>\alpha$. Due 
to $\gamma(0)=v_{1}=0$ and the continuity of $\gamma$, there exists $t_{\gamma} \in(0,1)$ such that $\left\|\gamma\left(t_{\gamma}\right)\right\|=\alpha$. Consequently, for any $\lambda \in I$, there holds

$$
\max _{t \in[0,1]} \mathcal{J}_{b, \lambda}^{T}(\gamma(t)) \geq c_{0}>0
$$

which shows that $c_{\lambda} \geq c_{0}$.

Lemma 3.3. Suppose that $\left(\mathrm{f}_{1}\right)-\left(\mathrm{f}_{3}\right)$ hold. Then for any $\lambda \in I$ and $b<1 /\left(8 T^{2}\right)$, each bounded $(P S)$ sequence of the functional $\mathcal{J}_{b, \lambda}^{T}$ at the level $c_{\lambda}$ admits a convergent subsequence in $E$.

Proof. Let $\lambda \in I$, suppose that $\left\{u_{n}\right\} \subset E$ is a bounded (PS) sequence of the functional $\mathcal{J}_{b, \lambda}^{T}$ at the level $c_{\lambda}$, namely

$$
\left\{u_{n}\right\} \text { is bounded and } \mathcal{J}_{b, \lambda}^{T}\left(u_{n}\right) \rightarrow c_{\lambda} ; \quad\left(\mathcal{J}_{b, \lambda}^{T}\right)^{\prime}\left(u_{n}\right) \rightarrow 0 \text { in } E^{-1} \text {. }
$$

Subject to a subsequence, we can assume that there exists $u$ in $E$ such that

$$
\begin{array}{ll}
u_{n} \rightarrow u & \text { in } E \\
u_{n} \rightarrow u & \text { on } L^{s}\left(\mathbb{R}^{N}\right), \forall s \in\left(2,2_{* *}\right), \\
u_{n} \rightarrow u & \text { a.e. on } \mathbb{R}^{N} .
\end{array}
$$

Thus, in view of the definition $\mathcal{J}_{b, \lambda}^{T}$, one has

$$
\begin{aligned}
o(1)= & \left\langle\left(\mathcal{J}_{b, \lambda}^{T}\right)^{\prime}\left(u_{n}\right), u_{n}-u\right\rangle \\
= & {\left[1+\frac{b}{2 T^{2}} \psi^{\prime}\left(\frac{\left\|u_{n}\right\|^{2}}{T^{2}}\right)\left(\int_{\mathbb{R}^{N}}\left|\nabla u_{n}\right|^{2} d x\right)^{2}\right]\left(u_{n}, u_{n}-u\right) } \\
& +b \psi\left(\frac{\left\|u_{n}\right\|^{2}}{T^{2}}\right) \int_{\mathbb{R}^{N}}\left|\nabla u_{n}\right|^{2} d x \int_{\mathbb{R}^{N}} \nabla u_{n} \nabla\left(u_{n}-u\right) d x \\
& -\lambda \int_{\mathbb{R}^{N}} f\left(u_{n}\right)\left(u_{n}-u\right) d x .
\end{aligned}
$$

Because $\left(\mathrm{f}_{1}\right)$ and $\left(\mathrm{f}_{2}\right)$, for any $\varepsilon>0$, there exists $C_{\varepsilon}>0$ such that

$$
|f(t)| \leq \varepsilon|t|+\varepsilon|t|^{2_{* *}-1}+C_{\varepsilon}|t|^{k_{0}-1}, \quad \forall t \in \mathbb{R}_{+},
$$

where $k_{0} \in\left(2,2_{* *}\right)$. It follows from Hölder inequality and 2.1 that

$$
\begin{aligned}
\left|\int_{\mathbb{R}^{N}} f\left(u_{n}\right)\left(u_{n}-u\right) d x\right| \leq & \varepsilon \int_{\mathbb{R}^{N}}\left|u_{n}\right|\left|u_{n}-u\right| d x+\varepsilon \int_{\mathbb{R}^{N}}\left|u_{n}\right|^{2_{* *}-1}\left|u_{n}-u\right| d x \\
& +C_{\varepsilon} \int_{\mathbb{R}^{N}}\left|u_{n}\right|^{k_{0}-1}\left|u_{n}-u\right| d x \\
\leq & \varepsilon\left\|u_{n}\right\|_{2}\left\|u_{n}-u\right\|_{2}+\varepsilon\left\|u_{n}\right\|_{2_{* *}^{2 * *}}^{2_{2^{*}}-1}\left\|u_{n}-u\right\|_{2_{* *}}
\end{aligned}
$$




$$
\begin{aligned}
& +C_{\varepsilon}\left\|u_{n}\right\|_{k_{0}}^{k_{0}-1}\left\|u_{n}-u\right\|_{k_{0}} \\
\leq & \varepsilon \gamma_{2}^{2}\left\|u_{n}\right\|\left\|u_{n}-u\right\|+\varepsilon \gamma_{2_{* *}^{* *}}^{2 *}\left\|u_{n}\right\|^{2_{* *}-1}\left\|u_{n}-u\right\| \\
& +C_{\varepsilon}\left\|u_{n}\right\|_{k_{0}}^{k_{0}-1}\left\|u_{n}-u\right\|_{k_{0}} \\
\rightarrow & 0 \quad \text { as } n \rightarrow+\infty .
\end{aligned}
$$

It is clear that

$$
b \psi\left(\frac{\left\|u_{n}\right\|^{2}}{T^{2}}\right) \int_{\mathbb{R}^{N}}\left|\nabla u_{n}\right|^{2} d x \int_{\mathbb{R}^{N}} \nabla u_{n} \nabla\left(u_{n}-u\right) d x \rightarrow 0 .
$$

What's more, since $b<1 /\left(8 T^{2}\right)$, one has

$$
\begin{aligned}
\frac{b}{2 T^{2}}\left|\psi^{\prime}\left(\frac{\left\|u_{n}\right\|^{2}}{T^{2}}\right)\right|\left(\int_{\mathbb{R}^{N}}\left|\nabla u_{n}\right|^{2} d x\right)^{2} & \leq \frac{b}{2 a^{2} T^{2}}\left|\psi^{\prime}\left(\frac{\left\|u_{n}\right\|^{2}}{T^{2}}\right)\right|\left\|u_{n}\right\|^{4} \\
& \leq \frac{4 b T^{2}}{a^{2}}<\frac{1}{2 a^{2}}
\end{aligned}
$$

Hence,

$$
\left[1+\frac{b}{2 T^{2}} \psi^{\prime}\left(\frac{\left\|u_{n}\right\|^{2}}{T^{2}}\right)\left(\int_{\mathbb{R}^{N}}\left|\nabla u_{n}\right|^{2} d x\right)^{2}\right]\left(u_{n}, u_{n}-u\right) \rightarrow 0 \quad \text { as } n \rightarrow+\infty,
$$

which implies that $u_{n} \rightarrow u$ in $E$.

Lemma 3.4. Suppose that $\left(\mathrm{f}_{1}\right)-\left(\mathrm{f}_{3}\right)$ hold. If $b<1 /\left(8 T^{2}\right)$, for almost every $\lambda \in I$, there exists $u_{\lambda} \in E \backslash\{0\}$ such that $\left(\mathcal{J}_{b, \lambda}^{T}\right)^{\prime}\left(u_{\lambda}\right)=0$ and $\mathcal{J}_{b, \lambda}^{T}\left(u_{\lambda}\right)=c_{\lambda}$.

Proof. The result follows immediately from Proposition 2.1 and Lemma 3.3 .

Lemma 3.5. Let $b<1 /\left(8 T^{2}\right)$. Suppose that $u_{n}$ is a critical point of the function $\mathcal{J}_{b, \lambda_{n}}^{T}$ at level $c_{\lambda_{n}}$. Then there exist $T>0$ and $b_{0} \in\left(0,1 /\left(8 T^{2}\right)\right)$ such that for all $b \in\left[0, b_{0}\right)$, up to a subsequence, $\left\|u_{n}\right\| \leq T$.

Proof. By Lemma 3.4, there exists a sequence $\left\{\lambda_{n}\right\} \subset I$ and $\left\{u_{n}\right\} \subset E$ such that

$$
\lambda_{n} \rightarrow 1^{-}, \quad \mathcal{J}_{b, \lambda_{n}}^{T}\left(u_{n}\right)=c_{\lambda_{n}} \quad \text { and } \quad\left(\mathcal{J}_{b, \lambda_{n}}^{T}\right)^{\prime}\left(u_{n}\right)=0 \quad \text { as } n \rightarrow+\infty .
$$

On the one hand, due to Lemma 2.2 and $\left(\mathcal{J}_{b, \lambda_{n}}^{T}\right)^{\prime}\left(u_{n}\right)=0$, one has

$$
\begin{aligned}
& {\left[(N-4) \int_{\mathbb{R}^{N}}\left|\Delta u_{n}\right|^{2} d x+a(N-2) \int_{\mathbb{R}^{N}}\left|\nabla u_{n}\right|^{2} d x+c N \int_{\mathbb{R}^{N}} u_{n}^{2} d x\right]} \\
& \times\left[\frac{1}{2}+\frac{b}{4 T^{2}} \psi^{\prime}\left(\frac{\left\|u_{n}\right\|^{2}}{T^{2}}\right)\left(\int_{\mathbb{R}^{N}}\left|\nabla u_{n}\right|^{2} d x\right)^{2}\right] \\
& +\frac{b(2 N-4)}{4} \psi\left(\frac{\left\|u_{n}\right\|^{2}}{T^{2}}\right)\left(\int_{\mathbb{R}^{N}}\left|\nabla u_{n}\right|^{2} d x\right)^{2} \\
& =\lambda_{n} N \int_{\mathbb{R}^{N}} F\left(u_{n}\right) d x .
\end{aligned}
$$


On the other hand, since $\mathcal{J}_{b, \lambda_{n}}^{T}\left(u_{n}\right)=c_{\lambda_{n}}$, we get

$$
\frac{1}{2} N\left\|u_{n}\right\|^{2}+\frac{b}{4} N h_{T}\left(u_{n}\right)\left(\int_{\mathbb{R}^{N}}\left|\nabla u_{n}\right|^{2} d x\right)^{2}-\lambda_{n} N \int_{\mathbb{R}^{N}} F\left(u_{n}\right) d x=N c_{\lambda_{n}} .
$$

Combing (3.4) with (3.5) we obtain

$$
\begin{aligned}
& \left(2 \int_{\mathbb{R}^{N}}\left|\Delta u_{n}\right|^{2} d x+a \int_{\mathbb{R}^{N}}\left|\nabla u_{n}\right|^{2} d x\right) \\
\leq & {\left[2+\frac{b}{T^{2}} \psi^{\prime}\left(\frac{\left\|u_{n}\right\|^{2}}{T^{2}}\right)\left(\int_{\mathbb{R}^{N}}\left|\nabla u_{n}\right|^{2} d x\right)^{2}\right] \int_{\mathbb{R}^{N}}\left|\Delta u_{n}\right|^{2} d x } \\
& +a\left[1+\frac{b}{2 T^{2}} \psi^{\prime}\left(\frac{\left\|u_{n}\right\|^{2}}{T^{2}}\right)\left(\int_{\mathbb{R}^{N}}\left|\nabla u_{n}\right|^{2} d x\right)^{2}\right] \int_{\mathbb{R}^{N}}\left|\nabla u_{n}\right|^{2} d x \\
= & \frac{N\left\|u_{n}\right\|^{2}}{2}\left[1+\frac{b}{2 T^{2}} \psi^{\prime}\left(\frac{\left\|u_{n}\right\|^{2}}{T^{2}}\right)\left(\int_{\mathbb{R}^{N}}\left|\nabla u_{n}\right|^{2} d x\right)^{2}\right] \\
& +\frac{b(2 N-4)}{4} \psi\left(\frac{\left\|u_{n}\right\|^{2}}{T^{2}}\right)\left(\int_{\mathbb{R}^{N}}\left|\nabla u_{n}\right|^{2} d x\right)^{2}-\lambda_{n} N \int_{\mathbb{R}^{N}} F\left(u_{n}\right) d x \\
= & N c_{\lambda_{n}}+\frac{N b}{4 T^{2}} \psi^{\prime}\left(\frac{\left\|u_{n}\right\|^{2}}{T^{2}}\right)\left(\int_{\mathbb{R}^{N}}\left|\nabla u_{n}\right|^{2} d x\right)^{2}\left\|u_{n}\right\|^{2}+\frac{b(N-4)}{4} \psi\left(\frac{\left\|u_{n}\right\|^{2}}{T^{2}}\right)\left(\int_{\mathbb{R}^{N}}\left|\nabla u_{n}\right|^{2} d x\right)^{2} .
\end{aligned}
$$

Now we define a function $\zeta:[0,1] \rightarrow E$ by

$$
\zeta(0)=0 \quad \text { and } \quad \zeta(t)=v_{2}\left(\frac{x}{t}\right)=z\left(\frac{x}{t t_{0}}\right), \quad \forall t \in(0,1] .
$$

It is obvious that $\zeta \in \Gamma$, and we have

$$
\begin{aligned}
c_{\lambda_{n}} \leq & \max _{t \in[0,1]} \mathcal{J}_{b, \lambda_{n}}^{T}(\zeta(t)) \\
\leq & \max _{t \in[0,1]}\left\{\frac{1}{2}\|\zeta(t)\|^{2}+\frac{b}{4} \psi\left(\frac{\|\zeta(t)\|^{2}}{T^{2}}\right)\left(\int_{\mathbb{R}^{N}}|\nabla \zeta(t)|^{2} d x\right)^{2}-\sigma \int_{\mathbb{R}^{N}} F(\zeta(t)) d x\right\} \\
\leq & \max _{t \in[0,1]}\left\{\frac{1}{2} \int_{\mathbb{R}^{N}}\left[\left(t t_{0}\right)^{N-4}|\Delta z|^{2}+a\left(t t_{0}\right)^{N-2}|\nabla z|^{2}+c\left(t t_{0}\right)^{N} z^{2}\right] d x-\sigma\left(t t_{0}\right)^{N} \int_{\mathbb{R}^{N}} F(z) d x\right\} \\
& +\frac{b}{4} \max _{t \in[0,1]}\left\{\psi\left(\frac{\|\zeta(t)\|^{2}}{T^{2}}\right)\left(\int_{\mathbb{R}^{N}}|\nabla \zeta(t)|^{2} d x\right)^{2}\right\} \\
\leq & \max _{t \geq 0}\left\{\frac{1}{2} \int_{\mathbb{R}^{N}}\left[t^{N-4}|\Delta z|^{2}+a t^{N-2}|\nabla z|^{2}+c t^{N} z^{2}\right] d x-\sigma t^{N} \int_{\mathbb{R}^{N}} F(z) d x\right\} \\
& +\frac{b}{4} \max _{t \in[0,1]}\left\{\psi\left(\frac{\|\zeta(t)\|^{2}}{T^{2}}\right)\left(\int_{\mathbb{R}^{N}}|\nabla \zeta(t)|^{2} d x\right)^{2}\right\} \\
:= & K_{1}+b K_{2}(T) .
\end{aligned}
$$

Since $\psi\left(\|u\|^{2} / T^{2}\right)=0$ for $2 T^{2}<\|u\|^{2}$, from the definition of $K_{2}(T)$ in (3.6) one has

$$
\begin{gathered}
b K_{2}(T) \leq \frac{b T^{4}}{a^{2}} \\
\frac{N b}{4 T^{2}} \psi^{\prime}\left(\frac{\left\|u_{n}\right\|^{2}}{T^{2}}\right)\left(\int_{\mathbb{R}^{N}}\left|\nabla u_{n}\right|^{2} d x\right)^{2}\left\|u_{n}\right\|^{2} \leq \frac{4 b N T^{4}}{a^{2}}
\end{gathered}
$$


and

$$
\frac{b(N-4)}{4} \psi\left(\frac{\left\|u_{n}\right\|^{2}}{T^{2}}\right)\left(\int_{\mathbb{R}^{N}}\left|\nabla u_{n}\right|^{2} d x\right)^{2} \leq \frac{b(N-4) T^{4}}{a^{2}} .
$$

Then, we get

$$
\left(2 \int_{\mathbb{R}^{N}}\left|\Delta u_{n}\right|^{2} d x+a \int_{\mathbb{R}^{N}}\left|\nabla u_{n}\right|^{2} d x\right) \leq N K_{1}+\frac{5 b N T^{4}}{a^{2}}+\frac{b(N-4) T^{4}}{a^{2}} .
$$

Finally, it follows from $\left(f_{1}\right),\left(f_{2}\right)$ and $\left(\mathcal{J}_{b, \lambda_{n}}^{T}\right)^{\prime}\left(u_{n}\right)=0$ that

$$
\begin{aligned}
& {\left[1+\frac{b}{2 T^{2}} \psi^{\prime}\left(\frac{\left\|u_{n}\right\|^{2}}{T^{2}}\right)\left(\int_{\mathbb{R}^{N}}\left|\nabla u_{n}\right|^{2} d x\right)^{2}\right]\left\|u_{n}\right\|^{2}+b \psi\left(\frac{\left\|u_{n}\right\|^{2}}{T^{2}}\right)\left(\int_{\mathbb{R}^{N}}\left|\nabla u_{n}\right|^{2} d x\right)^{2} } \\
= & \lambda_{n} \int_{\mathbb{R}^{N}} f\left(u_{n}\right) u_{n} d x \\
\leq & \varepsilon \int_{\mathbb{R}^{N}}\left|u_{n}\right|^{2} d x+C_{\varepsilon} \int_{\mathbb{R}^{N}}\left|u_{n}\right|^{2 * *} d x,
\end{aligned}
$$

together with 2.1 that

$$
\begin{aligned}
\left\|u_{n}\right\|^{2} & \leq \varepsilon \gamma_{2}^{2}\left\|u_{n}\right\|^{2}+C_{\varepsilon} \int_{\mathbb{R}^{N}}\left|u_{n}\right|^{2 * *} d x-\frac{b}{2 T^{2}} \psi^{\prime}\left(\frac{\left\|u_{n}\right\|^{2}}{T^{2}}\right)\left(\int_{\mathbb{R}^{N}}\left|\nabla u_{n}\right|^{2} d x\right)^{2}\left\|u_{n}\right\|^{2} \\
& \leq \varepsilon \gamma_{2}^{2}\left\|u_{n}\right\|^{2}+C_{\varepsilon} \int_{\mathbb{R}^{N}}\left|u_{n}\right|^{2 * *} d x+\frac{8 b T^{4}}{a^{2}} \\
& \leq \varepsilon \gamma_{2}^{2}\left\|u_{n}\right\|^{2}+C_{3}\left(\int_{\mathbb{R}^{N}}\left|\nabla u_{n}\right|^{2} d x\right)^{2_{* *} / 2}+\frac{8 b T^{4}}{a^{2}} \\
& \leq \varepsilon \gamma_{2}^{2}\left\|u_{n}\right\|^{2}+C_{4}\left[\frac{N K_{1}}{a}+\frac{5 b N T^{4}}{a^{3}}+\frac{b(N-4) T^{4}}{a^{3}}\right]^{2 * * / 2}+\frac{8 b T^{4}}{a^{2}} .
\end{aligned}
$$

Choosing $\varepsilon=1 /\left(2 \gamma_{2}^{2}\right)$, there exists $C_{5}>0$ such that

$$
\left\|u_{n}\right\|^{2} \leq C_{5}\left[\frac{N K_{1}}{a}+\frac{5 b N T^{4}}{a^{3}}+\frac{b(N-4) T^{4}}{a^{3}}\right]^{2_{* *} / 2}+\frac{16 b T^{4}}{a^{2}} .
$$

On the contrary, there exists no subsequence of $\left\{u_{n}\right\}$ which is uniformly bounded by $T$. Without loss of generality, we may suppose that $T<\left\|u_{n}\right\|, \forall n \in \mathbb{N}$. Then, by (3.7), we have

$$
T^{2}<\left\|u_{n}\right\|^{2} \leq C_{5}\left[\frac{N K_{1}}{a}+\frac{5 b N T^{4}}{a^{3}}+\frac{b(N-4) T^{4}}{a^{3}}\right]^{2 * * / 2}+\frac{16 b T^{4}}{a^{2}} .
$$

We can find $T_{*}>0$ such that

$$
1+C_{5}\left(\frac{N K_{1}}{a}\right)^{2 * * / 2} \leq T_{*}^{2}
$$


and $b_{0} \in\left(0,1 /\left(8 T_{*}^{2}\right)\right)$ with

$$
C_{5}\left[\frac{N K_{1}}{a}+\frac{5 b N T_{*}^{4}}{a^{3}}+\frac{b(N-4) T_{*}^{4}}{a^{3}}\right]^{2 * * / 2}+\frac{16 b T_{*}^{4}}{a^{2}} \leq C_{5}\left(\frac{N K_{1}}{a}\right)^{2_{* *} / 2}+1 .
$$

Thus, for any $b \in\left[0, b_{0}\right)$, it follows from $\left.(3.8)-3.10\right)$ that

$$
\begin{aligned}
C_{5}\left(\frac{N K_{1}}{a}\right)^{2 * * / 2}+1 & <C_{5}\left[\frac{N K_{1}}{a}+\frac{5 b_{0} N T_{*}^{4}}{a^{3}}+\frac{b_{0}(N-4) T_{*}^{4}}{a^{3}}\right]^{2 * * / 2}+\frac{16 b_{0} T_{*}^{4}}{a^{2}} \\
& \leq C_{5}\left(\frac{N K_{1}}{a}\right)^{2 * * / 2}+1,
\end{aligned}
$$

which is a contradiction. Then we complete the proof.

Now we are in the position to complete proof of Theorem 1.1 .

Proof of Theorem 1.1. Let $T$ and $b_{0}$ be defined as in Lemma 3.5. Suppose that $u_{n}$ is a critical point for $\mathcal{J}_{b, \lambda_{n}}^{T}$ at the level $c_{\lambda_{n}}$. From Lemma 3.5 , we get that

$$
\left\|u_{n}\right\| \leq T
$$

Further, we can obtain that

$$
\begin{aligned}
\mathcal{J}_{b, \lambda_{n}}^{T}\left(u_{n}\right) & =\frac{1}{2}\left\|u_{n}\right\|^{2}+\frac{b}{4}\left(\int_{\mathbb{R}^{N}}\left|\nabla u_{n}\right|^{2} d x\right)^{2}-\lambda_{n} \int_{\mathbb{R}^{N}} F\left(u_{n}\right) d x \\
& =\mathcal{J}_{b}\left(u_{n}\right)+\left(1-\lambda_{n}\right) \int_{\mathbb{R}^{N}} F\left(u_{n}\right) d x .
\end{aligned}
$$

From $\lambda_{n} \rightarrow 1^{-}$, it shows that $\mathcal{J}_{b}\left(u_{n}\right) \rightarrow c_{1}$. Moreover, we also have

$$
\begin{aligned}
\left\langle\left(\mathcal{J}_{b, \lambda_{n}}^{T}\right)^{\prime}\left(u_{n}\right), v\right\rangle & =\left(u_{n}, v\right)+b \int_{\mathbb{R}^{N}}\left|\nabla u_{n}\right|^{2} d x \int_{\mathbb{R}^{N}} \nabla u_{n} \nabla v d x-\lambda \int_{\mathbb{R}^{N}} f\left(u_{n}\right) v d x \\
& =\left\langle\mathcal{J}_{b}^{\prime}\left(u_{n}\right), v\right\rangle+\left(1-\lambda_{n}\right) \int_{\mathbb{R}^{N}} f\left(u_{n}\right) v d x .
\end{aligned}
$$

Therefore, $\mathcal{J}_{b}^{\prime}\left(u_{n}\right) \rightarrow 0$. Then $\left\{u_{n}\right\}$ is a bounded (PS) sequence of $\mathcal{J}_{b}$. By the similar discuss as the proof of Lemma 3.3 , we can get that $\left\{u_{n}\right\}$ has a convergent subsequence. We may suppose that $u_{n} \rightarrow u$ in $E$. Thus, $\mathcal{J}_{b}^{\prime}(u)=0$. Moreover, by Lemma 3.2 .

$$
\mathcal{J}_{b}(u)=\lim _{n \rightarrow+\infty} \mathcal{J}_{b}\left(u_{n}\right)=\lim _{n \rightarrow+\infty} \mathcal{J}_{b, \lambda_{n}}^{T}\left(u_{n}\right) \geq c_{0}>0
$$

together with the condition $\left(\mathrm{f}_{1}\right)$, we get that $u$ is a nonnegative solution of the problem (1.1). 


\section{Acknowledgments}

The authors express their gratitude to the reviewer for careful reading and helpful suggestions which led to an improvement of the original manuscript. This paper is partially supported by the National Natural Science Foundation of China (No. 61873248) and the Funds for International Cooperation and Exchange of the National Natural Science Foundation of China (No. 61860206014).

\section{References}

[1] J. M. Ball, Initial-boundary value problems for an extensible beam, J. Math. Anal. Appl. 42 (1973), 61-90.

[2] H. M. Berger, A new approach to the analysis of large deflections of plates, J. Appl. Mech. 22 (1955), 465-472.

[3] A. Cabada and G. M. Figueiredo, A generalization of an extensible beam equation with critical growth in $\mathbb{R}^{N}$, Nonlinear Anal. Real World Appl. 20 (2014), 134-142.

[4] M. Ferrara, S. Khademloo and S. Heidarkhani, Multiplicity results for perturbed fourth-order Kirchhoff type elliptic problems, Appl. Math. Comput. 234 (2014), 316325.

[5] S. Heidarkhani, M. Ferrara and S. Khademloo, Nontrivial solutions for onedimensional fourth-order Kirchhoff-type equations, Mediterr. J. Math. 13 (2016), no. $1,217-236$.

[6] S. Heidarkhani, S. Khademloo and A. Solimaninia, Multiple solutions for a perturbed fourth-order Kirchhoff type elliptic problem, Port. Math. 71 (2014), no. 1, 39-61.

[7] S. Heidarkhani and A. Salari, Existence of three solutions for impulsive perturbed elastic beam fourth-order equations of Kirchhoff-type, Studia Sci. Math. Hungar. 54 (2017), no. 1, 119-140.

[8] L. Jeanjean, On the existence of bounded Palais-Smale sequences and application to a Landesman-Lazer-type problem set on $\mathbb{R}^{N}$, Proc. Roy. Soc. Edinburgh Sect. A 129 (1999), no. 4, 787-809.

[9] L. Jeanjean and S. Le Coz, An existence and stability result for standing waves of nonlinear Schrödinger equations, Adv. Differential Equations 11 (2006), no. 7, 813840. 
[10] H. Kikuchi, Existence and stability of standing waves for Schrödinger-Poisson-Slater equation, Adv. Nonlinear Stud. 7 (2007), no. 3, 403-437.

[11] S. Khoutir and H. Chen, Least energy sign-changing solutions for a class of fourth order Kirchhoff-type equations in $\mathbb{R}^{N}$, J. Appl. Math. Comput. 55 (2017), no. 1-2, 25-39.

[12] S. Liang and J. Zhang, Existence and multiplicity of solutions for fourth-order elliptic equations of Kirchhoff type with critical growth in $\mathbb{R}^{N}$, J. Math. Phys. 57 (2016), no. $11,111505,13$ pp.

[13] J. Liu, S. Chen and X. Wu, Existence and multiplicity of solutions for a class of fourth-order elliptic equations in $\mathbb{R}^{N}$, J. Math. Anal. Appl. 395 (2012), no. 2, 608615.

[14] T. F. Ma, Positive solutions for a nonlocal fourth order equation of Kirchhoff type, Discrete Contin. Dyn. Syst. 2007 (2007), 694-703.

[15] P. J. McKenna and W. Walter, Travelling waves in a suspension bridge, SIAM J. Appl, Math. 50 (1990), no. 3, 703-715.

[16] H. Song and C. Chen, Infinitely many solutions for Schrödinger-Kirchhoff-type fourthorder elliptic equations, Proc. Edinb. Math. Soc. (2) 60 (2017), no. 4, 1003-1020.

[17] Y. Song and S. Shi, Multiplicity of solutions for fourth-order elliptic equations of Kirchhoff type with critical exponent, J. Dyn. Control Syst. 23 (2017), no. 2, 375386.

[18] F. Wang, T. An and Y. An, Existence of solutions for fourth order elliptic equations of Kirchhoff type on $\mathbb{R}^{N}$, Electron. J. Qual. Theory Differ. Equ. 2014 (2014), no. 39, $11 \mathrm{pp}$.

[19] F. Wang, M. Avci and Y. An, Existence of solutions for fourth order elliptic equations of Kirchhoff type, J. Math. Anal. Appl. 409 (2014), no. 1, 140-146.

[20] Y. Wang and Y. Shen, Multiple and sign-changing solutions for a class of semilinear biharmonic equation, J. Differential Equations 246 (2009), no. 8, 3109-3125.

[21] L. Xu and H. Chen, Existence and multiplicity of solutions for fourth-order elliptic equations of Kirchhoff type via genus theory, Bound. Value Probl. 2014, (2014):212, 12 pp.

[22] _ Multiple solutions for the nonhomogeneous fourth order elliptic equations of kirchhoff-type, Taiwanese J. Math. 19 (2015), no. 4, 1215-1226. 
[23] Multiplicity results for fourth order elliptic equations of Kirchhoff-type, Acta Math. Sci. Ser. B (Engl. Ed.) 35 (2015), no. 5, 1067-1076.

[24] Y. Ye and C.-L. Tang, Infinitely many solutions for fourth-order elliptic equations, J. Math. Anal. Appl. 394 (2012), no. 2, 841-854.

[25] Y. Yin and X. Wu, High energy solutions and nontrivial solutions for fourth-order elliptic equations, J. Math. Anal. Appl. 375 (2011), no. 2, 699-705.

[26] J. Zhang, X. Tang and W. Zhang, Existence of multiple solutions of Kirchhoff type equation with sign-changing potential, Appl. Math. Comput. 242 (2014), 491-499.

[27] J. Zhang and Z. Wei, Infinitely many nontrivial solutions for a class of biharmonic equations via variant fountain theorems, Nonlinear Anal. 74 (2011), no. 18, 74747485.

[28] Q. Zhang and J. Huang, Positive solutions for p-Laplacian equations of Kirchhoff type problem with a parameter, Electron. J. Differential Equations 2017 (2017), no. 292, $11 \mathrm{pp}$.

[29] W. Zhang, B. Cheng, X. Tang and J. Zhang, Sign-changing solutions for fourth order elliptic equations with Kirchhoff-type, Commun. Pure Appl. Anal. 15 (2016), no. 6, 2161-2177.

[30] W. Zhang, X. Tang and J. Zhang, Infinitely many solutions for fourth-order elliptic equations with general potentials, J. Math. Anal. Appl. 407 (2013), no. 2, 359-368.

Jianping Huang and Qi Zhang

School of Mathematics and Statistics, Central South University, Changsha, Hunan 410083, P. R. China

E-mail address: huangjianping@csu.edu.cn, zq8910@csu.edu.cn 